\section{Novel Life Science Tensile Stage Integration with Cryo Dual-Beam FIB Technology}

\author{
Debra M. Sherman \\ Life Science Microscopy Facility, Purdue Univ., W. Lafayette, IN \\ dsherman@purdue.edu
}

Soft and hydrated samples present unique challenges when determining structural integrity. Mechanical strength may be a critical factor in evaluating the sample in question. For example, genetic manipulation can change the chemical composition of plant cell walls. One goal would be producing strong plants whose stems and leaves lend themselves to easy break down for the production of ethanol. Arabidopsis is an ideal test sample for cell wall manipulation since the genetic strains are so well documented. However, these plants are quite small and mechanically testing the integrity of the walls can be problematic in the laboratory setting.

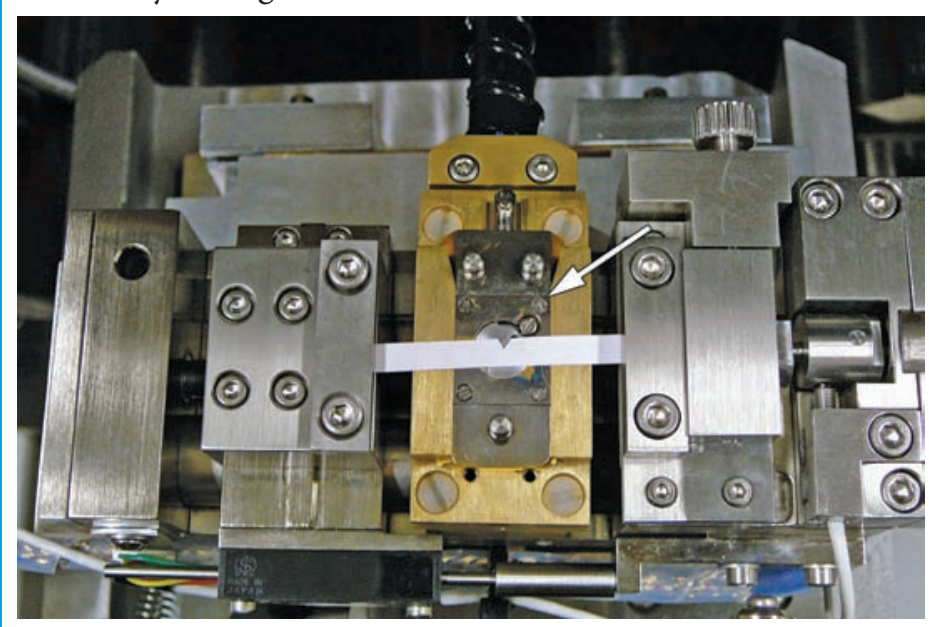

Figure 1: The tensile stage is shown with the cryo holder (arrow) inserted. The sample bridges the holder while undergoing tensile testing.

A tensile stage can be used to determine the force needed to produce tears in the plant material. But, there is then the problem of internal evaluation of the failure location. It became obvious to us that we needed to utilize new technology to test problems such as plant cell wall strength, radiation of micro-fractures through bone, and mechanical characteristics of synthetic materials for tissue replacement. We decided to approach this by designing a

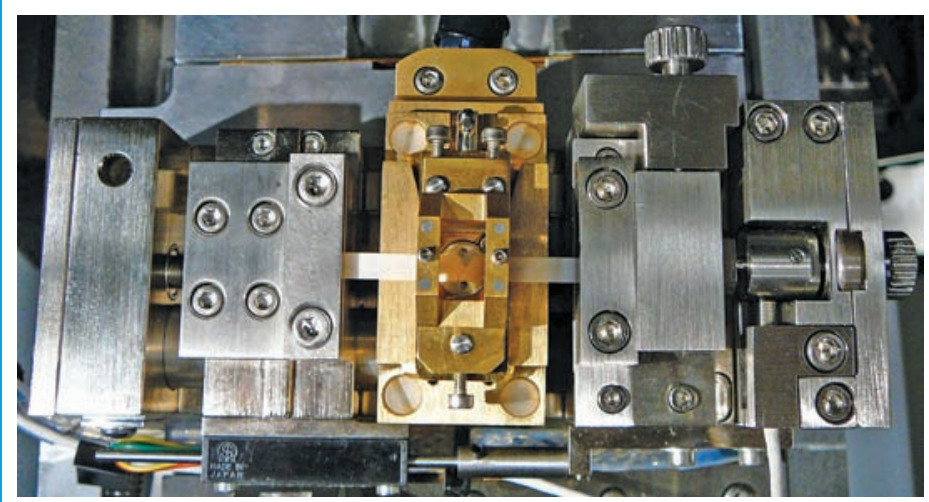

Figure 2: Strain is applied to a thin sliver of bone on the tensile stage. It is then clamped so that it can be cut free of the stage and frozen without release of tension.

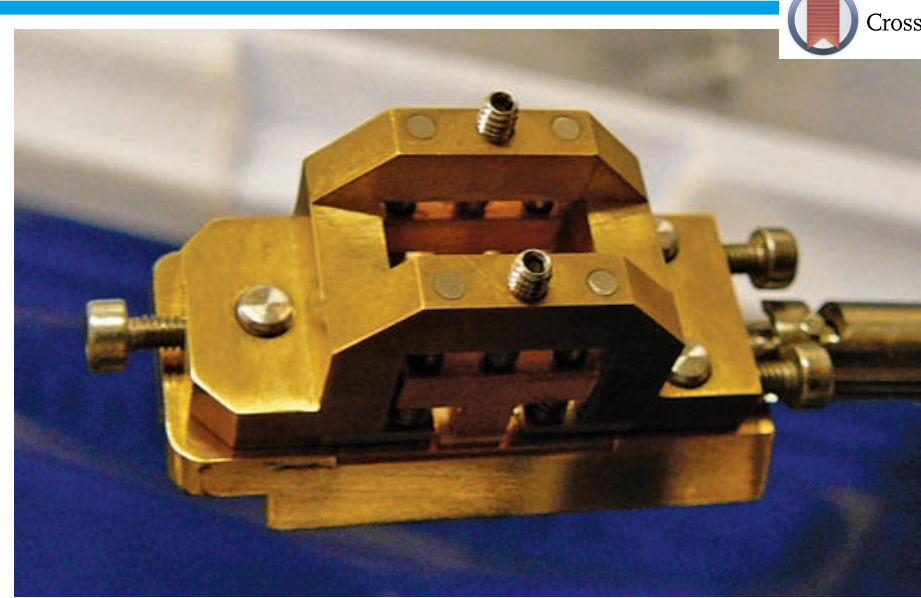

Figure 3: The sample, clamped to the cryo holder, is moved to the transfer chamber where the sample is immersed in liquid nitrogen and the clamp removed.

tensile stage capable of testing soft and hydrated materials. This would be followed by cryo focused ion beam (FIB) ablation in failure areas to further evaluate the physical effects of tensile forces within the sample. We required a uniquely designed tensile stage where the sample could be tested, clamped to a cryo holder, and frozen without release of tension. The sample would then be transferred into the cryo-preparation chamber while the microscope stage was cooled to cryo temperatures. It would then be moved onto the cryo stage for cryo dual-beam ablation and imaging.

An FEI Quant 3D FEG scanning electron microscope was the tool of choice for the project. This SEM has high vacuum, low vacuum, and ESEM modes with a field-emission gun to provide the required resolution. It also is a dual-beam instrument having both an ion beam for sample ablation (FIB) and an electron beam for imaging of the revealed surfaces. The Gatan Inc. Alto 2500 cryo unit was selected for cryo dual-beam use.

The tensile stage, designed by Gatan Inc. in consultation

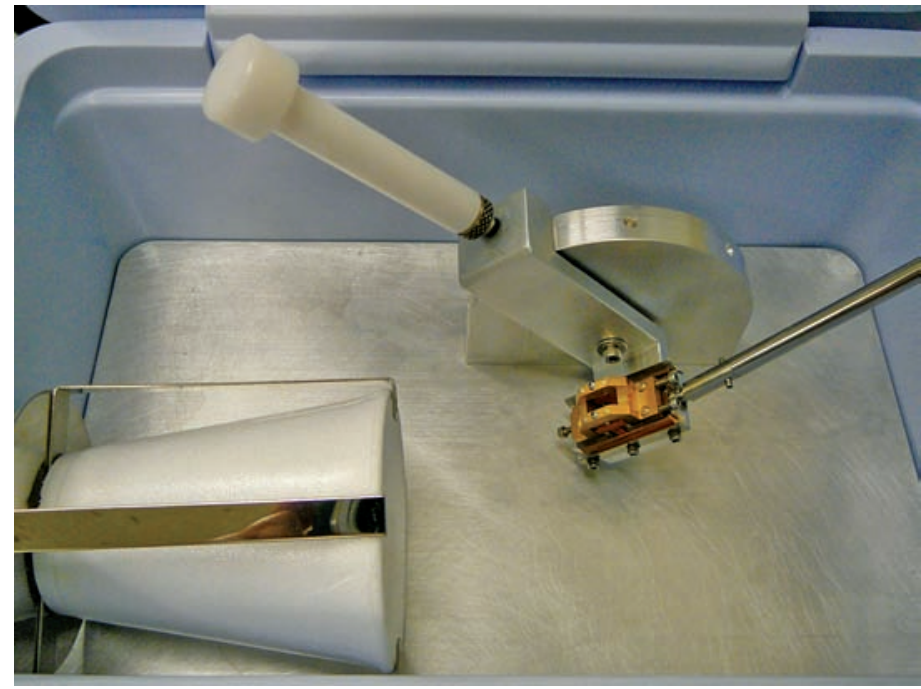

Figure 4: The transfer chamber consists of an insulated container, filled with liquid nitrogen, with a pivot mechanism. The sample holder can be frozen in liquid $\mathrm{N}_{2}$ and then tilted to easily remove the clamp. The holder is then inserted into a cup under the nitrogen and transferred to a pumping station where it is put under vacuum prior to transfer to the cryo unit preparation chamber for coating. 


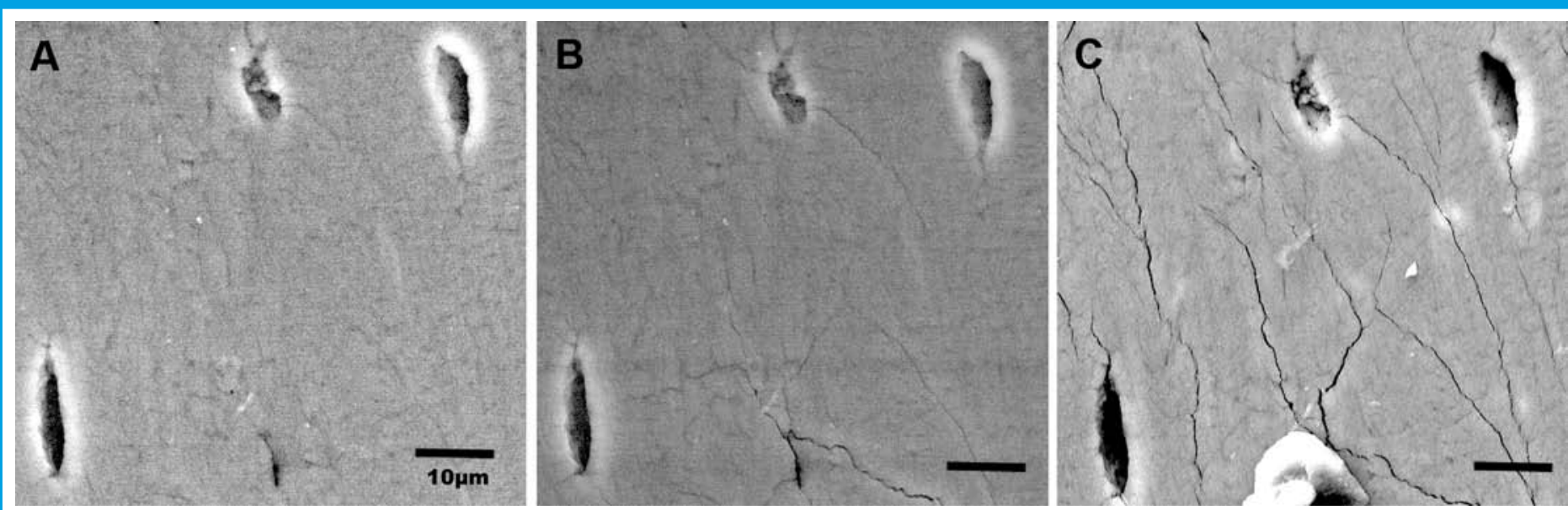

Figure5a-c: ESEM images show formation of microcracks in wet bone subjected to stretch at $60 \%$ humidity. (a) Initiation of stretch. (b) After applying $\sim 14$ newtons of force to the sample. The cracks are easily seen in high vacuum images of the frozen sample prior to dualbeam ablation (c). A piece of ice is present on the sample (lower edge).

with Purdue researchers, has a slot for insertion of a cryo holder just below the sample. (Figure 1). This holder is Peltier-cooled or heated as required. The sample is aligned over a slit in the cryo holder and observed with the electron beam during the tensile testing in low vacuum or ESEM mode.

A clamp is then applied to secure the sample (Figure 2), the sample is cut, and the holder removed from the tensile stage. A cryo-adhesive is applied to help secure the sample, which is then immersed in liquid nitrogen (Figure 3). The cryo holder can be rotated after freezing and prior to insertion into the cryo preparation chamber so that the FIB ablation can be oriented as desired in relation to direction of strain.

The clamp is removed and a transfer rod is attached in a transfer container designed at Purdue (Figure 4). The cryo holder is then moved to a nitrogen slush chamber where a vacuum is pulled prior to transferring the holder to the cryo preparation chamber chamber. There it can be sputter coated and held until needed. The preparation chamber can accommodate two cryo holders. Thus, two samples can be tested before the tensile stage is removed and replaced with the cryo stage, SEM pumped down, and the stage cooled to $\sim 120^{\circ} \mathrm{C}$. The sample is then transferred onto the stage and subjected to cryo dual-beam ablation and imaging.

Figure 5a-c illustrates the result of tensile stretch on wet bone. The bone is shown before (a) and after (b) stretch is applied resulting in microcrack formation. These cracks radiate out from the initial stress point in what appears to be a random array of cracks. The cracks are only easily visible when the sample is under tension. Once tension is relaxed the cracks close. This makes it very difficult to appreciate the full extent of the failure as shown by the intricate network of microcracks. Clamping the sample prior to removing it from the tensile stage and freezing preserves the sample morphology under tension. The cracks are more easily resolved under high vacuum cryo-imaging as seen in Figure $5 c$.

Both cryoSEM and ion beam ablation must be done in high vacuum. Therefore, the ability to convert from ESEM conditions to high vacuum cryo conditions quickly is essential.
Conversion, including cooling of the cryo stage to a working temperature of $-120^{\circ} \mathrm{C}$, can be done within 30 minutes utilizing the Gatan Alto 2500 cryo unit in the FEI Quanta 3D FEG DualBeam SEM.

\section{Acknowledgements:}

Umut Atakan Gurkan and Ji Hoon Jeon from the laboratory of Dr. Ozan Akkus, Assoc. Professsor of BioMedical Engineering, provided images $5 \mathrm{a}$ and $\mathrm{b}$ and expertise in using the tensile stage. Simon Galloway, Neil Othen, Marilyn Carey and Andrew Searle of Gatan Inc. were the principles in the design and production of the tensile stage.

\section{Why have 1,000 scientists in 25 countries selected Minus $\mathbf{K}^{\circ}$ vibration isolators?}

Because Minus $\mathrm{K}$ vibration isolation systems deliver $10 x$ to $100 x$ better performance than high-performance air systems, without air, and for air table prices.

\section{Visit www.minusk.com for more information..}

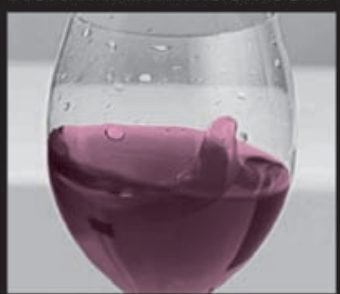

Without Minus K

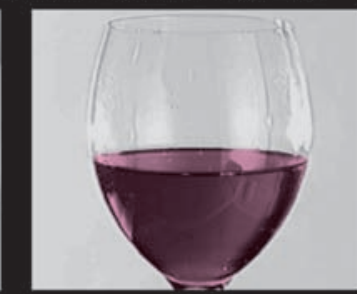

With Minus K

\section{VIBRATION ISOLATION BY:}

\section{minus $\mathbf{k}^{\circledR}$ TECHNOLOGY}

420 S. Hindry Ave., Unit E • Inglewood, CA 90301 USA Phone: $310-348-9656$ - Fax: $310-348-9638$ sales@minusk.com•www.minusk.com 